\title{
Pólipo del colon que genera un síndrome de ball valve. Reporte de un caso (con video)
}

\section{Ball Valve syndrome caused by a colon polyp. Case report (with video)}

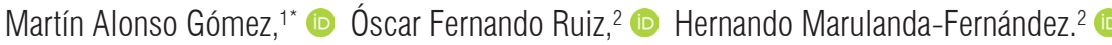

\author{
Gacceso abierto \\ Citación: \\ Gómez MA, Ruiz OF, Marulanda-Fernández H. \\ Pólipo del colon que genera un síndrome de \\ ball valve. Reporte de un caso (con video). Rev \\ Colomb Gastroenterol. 2020;35(4):519-521 \\ https://doi.org/10.22516/25007440.404 \\ Médico internista y gastroenterólogo, Unidad \\ de Gastroenterología y Ecoendoscopia (UGEC), \\ Hospital Universitario Nacional. Profesor de \\ Medicina, Universidad Nacional de Colombia. \\ Gastroenterólogo adscrito, Hospital Universitario \\ Fundación Santa Fe; Bogotá, Colombia. \\ Médico internista, gastroenterólogo, Universidad \\ Nacional de Colombia, Hospital Universitario \\ Nacional de Colombia; Bogotá, Colombia. \\ *Correspondencia: Martín Alonso Gómez \\ martinalonsogomezz@gmail.com \\ Fecha recibido: $\quad 30 / 01 / 18$ \\ Fecha aceptado: $30 / 03 / 18$ \\ (c) $(1) \Theta$
}

\begin{abstract}
Resumen
La obstrucción intestinal es una patología potencialmente letal y cuyo tratamiento, por lo general, es quirúrgico. Presentamos el caso de un paciente con dolor abdominal y clínica de obstrucción intestinal recurrente, en quien se documenta, durante una colonoscopia, un gran pólipo pediculado que causaba obstrucciones parciales por el fenómeno de ball valve.
\end{abstract}

\section{Palabras clave}

Pólipo, ball valve, obstrucción intestinal, polipectomía.

\section{Abstract}

Intestinal obstruction is a potentially lethal pathology, and its treatment is usually surgical. The following is the case of a patient with abdominal pain and recurrent intestinal obstruction, in whom a large pediculated polyp that caused partial obstruction by Ball valve effect was observed during a colonoscopy.

\section{Keywords}

Polyp, Ball Valve, Bowel obstruction, Polypectomy.

\section{INTRODUCCIÓN}

El síndrome de ball valve fue descrito por primera vez en 1946 por Hobbs y Cohen (1). Desde entonces ha sido reconocido como una causa rara pero importante de dolor abdominal recurrente (2). Se presenta principalmente en el tracto digestivo superior (3) y compromete, en orden de frecuencia, el duodeno y el píloro por lesiones que se prolapsan (4). Su etiología es variable y puede estar relacionada con pólipos, tumores y lesiones subepiteliales benignas, como los lipomas de gran tamaño (5). Si bien la afección colónica ha sido referida, se considera muy poco frecuente (6). Para nuestro conocimiento, este es el primer caso reportado en Colombia.

\section{CASO CLÍNICO}

Paciente de 42 años con múltiples ingresos hospitalarios a causa de un cuadro clínico de 3 meses de evolución, consistente en dolor abdominal, vómito, pérdida de peso y altera- 
ción del hábito intestinal, por lo cual presentó episodios de estreñimiento alternados por diarrea. Durante sus últimos ingresos a urgencias se consignó en la historia clínica el diagnóstico de obstrucción intestinal. Sin embargo, con una rápida respuesta al manejo médico, las pruebas bioquímicas fueron normales, mientras que la radiografía $(\mathrm{Rx})$ de abdomen simple determinó la existencia de un íleo inespecífico, y la tomografía axial computarizada (TAC) de abdomen permitió observar la presencia de un engrosamiento inespecífico del colon sigmoide. Dada la persistencia de los síntomas, el servicio de cirugía general decidió hospitalizar y programar una laparoscopia diagnóstica.

Durante la hospitalización, el paciente presentó una agudización de los síntomas, que se agravó por una rectorragia. Por este motivo, se solicitó realizar una colonoscopia total, en la cual se observó un gran pólipo pediculado de $40 \mathrm{~mm}$, con un pedículo largo de aproximadamente $30 \mathrm{~mm}$ en el colon sigmoide, que mostró un prolapso causado por peristaltismo, lo que generó una obstrucción de la luz colónica (Figuras 1-4). La cabeza del pólipo estaba erosionada como una probable causa de rectorragia.

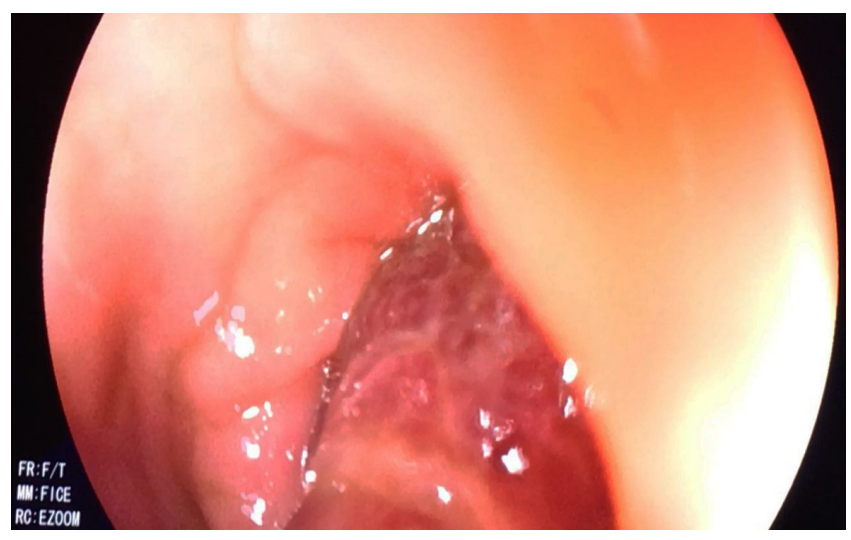

Figura 1. Gran pólipo pediculado del colon.

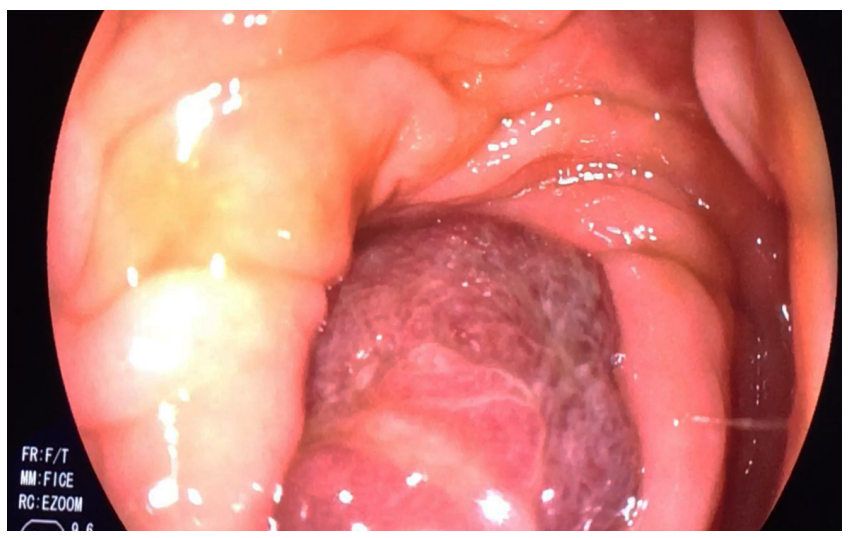

Figura 2. Pólipo del colon que ocluye en su totalidad la luz colónica.

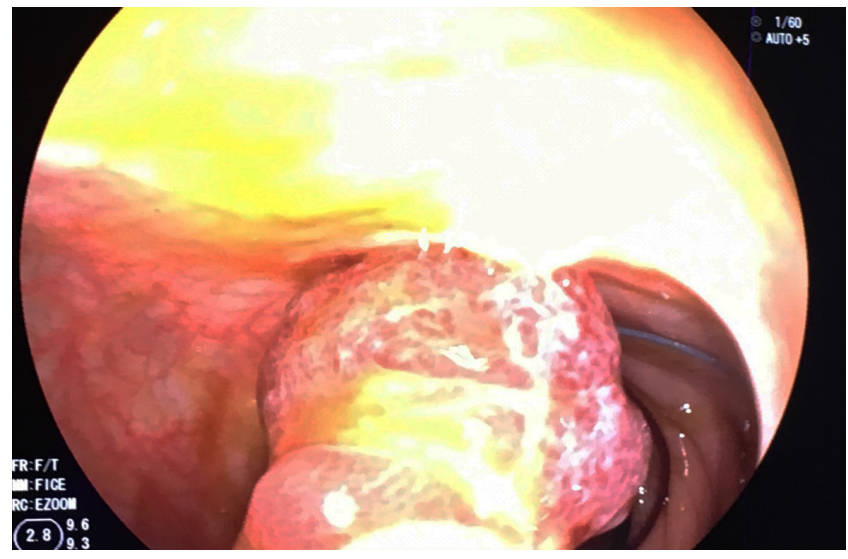

Figura 3. Paso del lazo hemostático (endoloop) previo a la polipectomía.

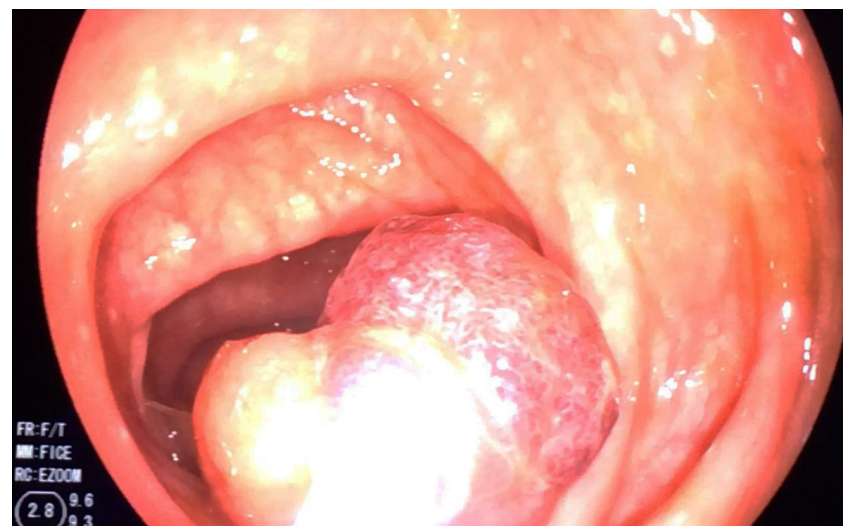

Figura 4. Recuperación del pólipo del colon resecado.

Se decidió entonces realizar una polipectomía endoscópica. Además, ante el tamaño del pedículo, se optó por insertar un lazo hemostático (endoloop). Luego de ello, se usó el asa de polipectomía y se resecó el pólipo (Video 1).

\section{Ver video}

Video 1. Síndrome de ball valve en colon. https://youtu.be/UsTxhTlJtUQ

En consecuencia, la evolución del paciente fue favorable, los síntomas se resolvieron y durante el seguimiento de más de 5 meses el individuó permaneció asintomático. El reporte de la patología reveló un pólipo adenomatoso.

\section{DISCUSIÓN}

El síndrome de ball valve es una complicación mecánica de las lesiones endoluminales de gran tamaño (7). Estas pueden generar fenómenos de pseudoobstrucción por 
prolapsos intermitentes facilitados por el peristaltismo, los cuales ocupan la luz de forma temporal (8). Los síntomas dependerán del lugar comprometido por la lesión.

Dado que se considera un fenómeno dinámico, el cuadro sintomático suele autolimitarse (9), motivo por el cual también tiende a cronificarse (4), a partir de repercusiones nutricionales importantes, pérdida de peso inexplicada y manifestaciones sintomáticas que fácilmente pueden confundirse con una patología de origen tumoral (10). Este escenario implica múltiples estudios e intervenciones (en algunos casos innecesarios), así como también repercusiones inaceptables sobre la calidad de vida del paciente (11).

El tratamiento endoscópico es ampliamente recomendado; sin embargo, según la naturaleza de la lesión, puede requerirse un manejo quirúrgico complementario (2).

\section{REFERENCIAS}

1. de la Plaza R, Picardo AL, Cuberes R, Jara A, MartínezPeñalver I, Villanueva MC, Medina M, Alías D, Osorio S, Pacheco E, Suárez A. Inflammatory fibroid polyps of the large intestine. Dig Dis Sci. 1999;44(9):1810-6. http://doi.org/10.1023/a:1018886421409

2. Macedo G, Lopes S, Albuquerque A. Ball valve syndrome: gastric polypectomy as a safe endoscopic treatment of a potentially troublesome condition. Gastrointest Endosc. 2012;76(5):1080-1. http://doi.org/10.1016/j.gie.2012.06.018

3. Akbulut S. Intussusception due to inflammatory fibroid polyp: a case report and comprehensive literature review. World J Gastroenterol. 2012;18(40):5745-5752. http://doi.org/10.3748/wjg.v18.i40.5745

4. Martins CR, Gamito É, Oliveira AP. Ball valve syndrome caused by a giant gastric Vanek's tumor. Rev Esp Enferm Dig. 2017;109(3):237-238. http://doi.org/10.17235/reed.2017.4526/2016

5. Sun CK, Yang KC, Liao CS. Endoscopic Management of Gastric Polyp with Outlet Obstruction without Polypectomy. Case Rep Gastroenterol. 2011;5(2):267-271. http://doi.org/10.1159/000328443
6. Kimura H, Yoshida T, Takahashi I. Ball valve syndrome. Gastrointest Endosc. 2003;58(1):99.

7. Sebastian S, Addley J, Crotty P, Buckley M. Giant gastric polyp. Gastrointest Endosc. 2004;59(3):398-9. http://doi.org/10.1016/s0016-5107(03)02595-1

8. Ozturk O, Koklu S, Sokmensuer C, Uner H. A giant benign polyp of the stomach. Gastroenterol Nurs. 2016;39(6):478-480. http://doi.org/10.1097/SGA.0000000000000239

9. Yriberry Ureña S, Vila Guitérrez S, Salazar Muente F. Polypectomy and endoscopic management of a giant gastric polyp. Rev Gastroenterol Perú. 2010;30(2):167-71.

10. Iso Y, Sawada T, Rokkaku K, Shimoda M, Kubota K. Ball-valve gastric tumor associated with anomalous junction of the pancreatico-biliary ductal system and a right-sided round ligament: report of a case. Surg Today. 2008;38(5):458-62. http://doi.org/10.1007/s00595-007-3635-0

11. Pinto-Pais T, Fernandes S, Proença L, Fernandes C, Ribeiro I, Sanches A, Carvalho J, Fraga J. A Large Gastric Inflammatory Fibroid Polyp. GE Port J Gastroenterol. 2015;22(2):61-64. http://doi.org/10.1016/j.jpge.2014.07.006 